\title{
Cosmic Star Formation History from Local Observations and an Outline for Galaxy Formation and Evolution
}

\author{
F.D.A. Hartwick \\ Department of Physics and Astronomy, \\ University of Victoria, Victoria, BC, Canada, V8W $3 P 6$
}

\begin{abstract}
The goal of this investigation is to reconstruct the cosmic star formation rate density history from local observations and in doing so to gain insight into how galaxies might have formed and evolved. A new chemical evolution model is described which accounts for the formation of globular clusters as well as the accompanying field stars. When this model is used in conjunction with the observed age metallicity relations for the clusters and with input which allows for the formation of the nearly universally observed bimodal distribution of globular clusters, star formation rates are obtained. By confining attention to a representative volume of the local universe, these rates allow a successful reconstruction of the Madau plot while complementary results similtaneously satisfy many local cosmological constraints. A physical framework for galaxy formation is presented which incorporates the results from this chemical evolution model and assumes an anisotropic collapse. In addition to providing the 'classical' halo, bulge and disk components, the model also predicts a new stellar halo component with peak $[\mathrm{Fe} / \mathrm{H}] \sim-0.8$ and disk-like angular momentum and allows for the formation of a thick disk as outlined by the group of metal rich globular clusters. Milky Way counterparts of the latter two components are identified.
\end{abstract}

Subject headings: Galaxy: formation — Galaxy: halo — globular clusters: general

\section{Introduction}

Observations of the luminosity functions of high redshift galaxies enable one to determine in situ the star formation rate density (SFRD) as a function of redshift. A plot of these two quantities has become known as a Madau diagram (Lilly et al. 1996, Madau et al. 1996, 1998). Locally, we observe the results of this early evolution in the form of old stars and globular 
clusters. Hence, given a model of chemical evolution from which the metallicity distribution functions (MDF's) are obtained, combined with the corresponding age-metallicity relations, we should be able to reconstruct the star formation history from these local observations and compare it to what is currently observed at high redshift.

A very preliminary investigation of this problem along these lines was given by Hartwick (1999). Since then, many more high redshift observations have become available (i.e. the Madau plot has been extended), the cosmological parameters have become better defined (providing a more reliable age-redshift relation), and age-metallicity relations for globular clusters have become available.

In this paper a new chemical evolution model is described which is designed to account for the formation of both the globular clusters and the accompanying suite of field stars. The model borrows from the classical works of galaxy formation (Eggen, Lynden-Bell, \& Sandage 1962, and Searle \& Zinn 1978) in that while undergoing large scale collapse, chemical evolution is assumed to proceed quiescently in protogalactic clumps which then collide as isolated units to form globular clusters, release stars already formed, and provide enriched and recyclable gas. The input to the model are the properties of two principal groups of globular clusters described below.

\section{Chemical Evolution and the Formation of Globular Clusters}

Possibly the most complete representative sample of the earliest local historical record is the system of globular clusters. For the majority of the clusters, we know within varying degrees of uncertainty their chemical abundances, motions, positions, and their ages. Unfortunately we still do not know how they formed. Because the evolution of individual stars is the only known way of enriching the gas from which the clusters formed, there must be a population of stars which formed with a distribution of heavy element abundance ending at that of a particular cluster. Further, because we know the heavy element distribution among clusters from observations, given a theory of chemical evolution we can calculate the abundance distribution of these accompanying stars. If as we shall assume below, that the clusters are formed in the shocked gas caused by collisions between isolated chemically evolving clumps for example, when the cluster is formed any remaining gas will lose energy but not angular momentum while the previously formed stars and accompanying dark matter will continue their dynamical evolution relatively unscathed.

As discussed below there appear to be two groups of clusters in the Galaxy. For convenience we shall refer to them as the blue and red groups. The blue group is relatively 
metal poor, has low angular momentum, is spatially extended far out into the halo, and is generally nearly as old as the universe itself. The red group is relatively metal rich, shows rapid rotation at all radii, and possesses a disk like spatial distribution with a concentration towards the Galactic center. The diversity between the two groups suggests separate stages of formation and in our chemical evolution model each group is evolved separately. In the following model, the main thrust is consideration of the effects of globular cluster formation on chemical evolution and not the formation mechanism itself. For the latter subject we refer to the more sophisticated modelling of Cote et al. (2000,2002), Beasley et al. (2002), and Kravtsov \& Gnedin (2003). Like the above cited works, ours is a bottom-up picture with the formation mechanism most resembling that for the metal rich clusters in the Beasley et al. work (i.e. collisions between gas rich clumps). For simplicity we assume that all clusters are formed in this way. In order to account for the bimodality, we argue that the collapse was anisotropic.

The objective of this work is to reconstruct the cosmic star formation history from local observations and in doing so to gain further insight into how galaxies might have formed and evolved. As will be shown, this reconstruction is successful, comparing favorably with that deduced from high redshift observations (the Madau plot). This implies that our model provides a scenario for the chemical evolution of a representative sample of the universe. Further, a proposed physical framework incorporates the results from this chemical evolution model and predicts a major new stellar halo component, which we identify in the Milky Way with the thick disk.

\subsection{The Apparent Universality of Globular Cluster Bimodality}

Classical globular clusters like M92 belong to a group of relatively blue, metal poor clusters which are old and are found in the outer halo of the Galaxy. It was early work by Becker (1950), Baade (1958), Morgan (1959) and Kinman (1959) which directed attention to the disk-like spatial distribution of a second group of globular clusters which are redder, more metal rich, some what younger, and concentrated towards the center of the Galaxy. An important further difference is that whereas the metal poor group shows very little rotation about the Galactic center, the metal rich group shows significant rotation at all Galactocentric radii (Cote, 1999, Zinn 1985). A clear separation of the two groups shows in the frequency histogram of $[\mathrm{Fe} / \mathrm{H}]$ values for 133 Milky Way globular clusters in Fig. 8 of Cote (1999) along with best fit Gaussians for each group.

Recent spectroscopic studies of the globular clusters in M31 by Perrett et al. (2002), and earlier work by Barmby et al. (2000) and Huchra et al. (1991) also show a bimodal distribution 
in $[\mathrm{Fe} / \mathrm{H}]$ with Gaussian parameters similar to those of the Milky Way. In fact, the bimodality of globular cluster populations appears to be common in more distant luminous galaxies where it is usually observed as a bimodality in color distribution (e.g. Ashman \& Zepf 1998, Gebhardt \& Kissler-Patig 1999, Forbes et al. 2001, Kundu \& Whitmore 2001, Goudfrooij et al. 2003, and references therein).

\subsection{A New Chemical Evolution Model to Accommodate Globular Cluster Formation}

The main ingredients of the model presented in this paper are the globular clusters. Unfortunately, it is still not understood in detail how these clusters are formed. One interesting possibility, outlined by McCrea (1982), suggests that clusters form in the compressed region between colliding streams or clumps of gas. Gunn (1980) uses a similar argument as a means to attain globular cluster sized Jeans masses at temperatures of a few thousand degrees. Some observational evidence for such a formation mechanism also exists (Schweitzer 1987). Given our present lack of understanding of how the clusters formed, we will assume the following phenomenological scenario. The starting point consists of a large number of protogalactic clumps (baryons inside dark matter halos) which, although spatially isolated, chemically evolve as in the simple mass loss model (a 'quiescent' mode of star formation). The gas in these clumps is assumed to have been initially enriched in a previous Population III phase. When these clumps collide, globular clusters are assumed to form with the metal abundance of the gas in the colliding clumps (a 'burst' mode of star formation). By design, the mass in cluster stars is small compared to the total mass of all stars. After most collisions, quiescent star formation in each colliding clump ceases and the remaining gas, having lost energy but not angular momentum in the collision, falls towards the center of the protogalaxy to be recycled later. The stars previously formed quiescently (now considered field stars) and the dark matter continue their previous dynamical evolution, while the newly formed clusters are not expected to have the same kinematics or spatial distribution as these field stars. Relatively few collisions could also lead to cluster formation and coalescence of the interacting clumps to form a satellite galaxy.

The context of the new chemical evolution model is as follows. In the well known mass

loss model of chemical evolution described below one starts with a mass of gas which is slowly turned into stars while enriched gas is lost at a rate proportional to the star formation rate. The constant of proportionality determines the 'effective' yield. The evolution ends when the gas is exhausted due to mass loss. In the new model we imagine a large number of 'chemical evolution boxes' which individually evolve as above but may now collide with a neighbor. 
This collision is assumed to create a globular cluster and halt the star formation in both colliding clumps. As discussed above, the colliding gas dissipates energy and falls to the center or as close to the center as the conserved angular momentum will allow. Meanwhile the stars and dark matter pass through and continue their previous dynamical evolution. In addition to the original mass loss process which can be thought of as 'supernova driven' and hence is assumed to remove gas from further star formation, we now have a second mass loss process-the collisions-which cause the gas to lose energy and thus be available for later recycling. Because the clusters are assumed to form in collisions and because the abundance distribution of each group can be represented by a normal distribution, in the model we make the collision rate normally distributed in $[\mathrm{Fe} / \mathrm{H}]$ (i.e. the role of globular cluster formation is to effect a sudden cessation of star formation by removal of the remaining gas from individual clumps in a Gaussian distributed manner in $[\mathrm{Fe} / \mathrm{H}])$.

Quantitatively, in the simple mass loss model of chemical evolution (Hartwick 1976, see also Pagel 1997, p.234 and Binney \& Merrifield 1998, p.308) the three components are the mass in stars, $\mathrm{M}_{s}$, the mass in remaining gas, $\mathrm{M}_{g}$, and the mass lost due to star formation, here denoted $\mathrm{M}_{W \text { HIM }}$ in anticipation of later discussion. As the chemical evolution proceeds, the gas is gradually depleted and similtaneously enriched in heavy elements due to ongoing star formation, while also being lost at a rate proportional to the star formation rate. The equations governing each component for an initial mass of gas $\mathrm{M}_{t}$ of metallicity $\mathrm{Z}_{0}$, can be written as

$$
\begin{gathered}
\frac{d M_{s}}{d \log Z}=\ln 10 \times Z \times M_{g} / p \\
M_{g}=M_{t} \times \exp \left(-\left(Z-Z_{0}\right)(1+c) / p\right) \\
M_{W H I M}=c \times M_{s}
\end{gathered}
$$

where $\mathrm{M}_{s}\left(\mathrm{Z}_{0}\right)=0$ and $\mathrm{M}_{g}\left(\mathrm{Z}_{0}\right)=\mathrm{M}_{t}$, $\mathrm{p}$ is the yield of heavy elements and $\mathrm{c}$ is the ratio of mass loss rate to star formation rate and also determines the 'effective' yield (i.e. $p_{\text {eff }}=p /(1+c)$ ).

In our new model, it is assumed that the collisions between clumps which halt the chemical evolution are distributed normally with mean $\log \mathrm{Z}_{c}$ and standard deviation $\sigma$. As the collisions occur, the mass in surviving clumps diminishes. This surviving clump mass with metallicity greater than $\log \mathrm{Z}$ is $\mathrm{M}_{t} \times \mathrm{f}(>\log \mathrm{Z})$ where the initial mass of gas in all clumps with metallicity $\mathrm{Z}_{0}$ is $\mathrm{M}_{t}$ and

$$
f(>\log Z) \simeq \frac{1}{\sqrt{2 \pi} \sigma} \int_{\log Z}^{\infty} \exp \left(-\frac{\left(\log Z^{\prime}-\log Z_{c}\right)^{2}}{2 \sigma^{2}}\right) d \log Z^{\prime}
$$

which is valid when $\mathrm{Z}_{0} \ll \mathrm{Z}_{c}$. Note that for computing purposes the right hand side of (4) is one half of the complementary error function i.e. $f(>\log Z)=0.5 \times \operatorname{erfc}\left(\frac{\left(\log Z-\log Z_{c}\right)}{\sqrt{2} \sigma}\right)$. 
By replacing $\mathrm{M}_{t}$ with $\mathrm{M}_{t} \times \mathrm{f}$, equations (1) and (2) can be written as

$$
\begin{gathered}
\frac{d M_{s}}{d \log Z}=\ln 10 \times Z \times M_{g} / p \\
M_{g}=M_{t} \times 0.5 \times \operatorname{erfc}\left(\frac{\left(\log Z-\log Z_{c}\right)}{\sqrt{2} \sigma}\right) \times \exp \left(-\left(Z-Z_{0}\right)(1+c) / p\right)
\end{gathered}
$$

Note that equations (1) \& (3) remain the same. (Equation (1) remains the same because the metallicity of the gas lost by collisions is the same as that in the clumps at the time of the collision). Because we now have an additional mass loss component, we define a new variable $\mathrm{M}_{m l}$, which represents the gas lost when the clumps collide. Whereas $\mathrm{M}_{W H I M}$ is considered here to be lost to the 'warm hot intergalactic medium', the new $\mathrm{M}_{m l}$ component, having lost energy in the collision, is assumed to fall to the center of the proto-galaxy to be recycled. Figuratively, an individual classical chemical evolution 'box' is totally purged by having gas drop out of the bottom on collision as well as having been blown continuously out of the top as a result of previous ongoing star formation! The equations governing the evolution of these two mass loss components are

$$
M_{W H I M}=c \times M_{s}
$$

and

$$
\frac{d M_{m l}}{d \log Z}=M_{t} \times \exp \left(-\left(Z-Z_{0}\right)(1+c) / p\right) \times \frac{1}{\sqrt{2 \pi} \sigma} \times \exp \left(-\frac{\left(\log Z-\log Z_{c}\right)^{2}}{2 \sigma^{2}}\right)
$$

We limit the number of parameters by letting $Z_{c}$ define the effective yield, i.e.

$$
c=\left(p / Z_{c}-1\right)
$$

Equation (5) represents the MDF of all stars formed (including those in globular clusters). We have not attempted to model globular cluster formation. It is assumed that the clusters are formed in bursts during collisions between clumps. We do attempt to make the chemical evolution consistent by ensuring that stars are formed which, if assembled into a cluster system, would have the requisite mass and (by design) the appropriate Gaussian abundance distribution. To this end we express the amplitude of the cluster MDF as the product of the total baryonic mass $\left(\mathrm{M}_{t}\right)$ (e.g. McLaughlin, 1999) and an efficiency factor $\eta$ so that

$$
\frac{d M_{G C}}{d \log Z}=\eta M_{t} \frac{1}{\sqrt{2 \pi} \sigma} \exp \left(-\frac{\left(\log Z-\log Z_{c}\right)^{2}}{2 \sigma^{2}}\right),
$$

where $\eta=0.00068$. This value of $\eta$ was chosen in order to ensure the assembly of $\sim 100$ metal poor (blue) clusters in the model to be discussed later. For the parameters adopted in 
the model to follow, all but the most metal rich $3 \%$ of the the blue clusters and $99.8 \%$ of the same number of metal rich (red) clusters can be accommodated before the gas is exhausted. Since the $\mathrm{M}_{m l}$ component eventually gets turned into stars, the ratio of the integrated mass in clusters to that in stars (i.e. $\left(\mathrm{M}_{G C} /\left(\mathrm{M}_{s}+\mathrm{M}_{m l}-\mathrm{M}_{G C}\right)\right)$ is $\sim 0.001$ which is within a factor of two of observational determinations of the same quantity (e.g. McLaughlin, 1999).

We reiterate that in the context of this model the formation of globular clusters only determines the extent of the chemical evolution within individual clumps. It is the stars produced that enriched the gas from which the clusters formed and the enriched gas lost both due to star formation and collisions which are the dominant end products in this model and not the individual clusters.

Equations (5)-(10) along with boundary conditions $\mathrm{M}_{s}\left(Z_{0}\right)=0, \mathrm{M}_{g}\left(Z_{0}\right)=\mathrm{M}_{t}, \mathrm{M}_{W H I M}\left(Z_{0}\right)=$ $0, \mathrm{M}_{m l}\left(Z_{0}\right)=0$ and $\mathrm{M}_{G C}\left(Z_{0}\right)=0$ and parameters $Z_{c}, Z_{0}, \sigma$ and $\eta$ define the new chemical evolution model. Figure 1 shows the evolution of the independent quantities for an illustrative set of parameters. The parameters were chosen to provide observable separation of the four components, but it should be emphasized that the model was motivated such that $\log \mathrm{Z}_{c}$ and $\sigma$ are determined by a particular globular cluster abundance distribution. As a result of the loss of gas due to collisions, the peak in the accompanying stellar metal abundance distribution is found to be $\sim 0.25$ dex lower than the corresponding globular cluster peak $\left(\log \mathrm{Z}_{c}\right)$ for values of $\sigma$ of order $0.2-0.3$.

\subsection{Colliding Boxes and the Stellar Halo of M31}

Durrell et al. (2001) have probed the halo of M31 at a distance of $\sim 20 \mathrm{kpc}$ from the center and have determined the metallicity distribution function (MDF) photometrically. This study provides an excellent 'test' sample for the new model, since all stars are essentially at the same distance from the Milky Way and the photometry is sufficiently deep that there should be no serious observational selection effects. Their results are reproduced as the histogram in Figure 2, where for consistency their metallicity scale $[\mathrm{m} / \mathrm{H}]$ has been converted to $[\mathrm{Fe} / \mathrm{H}]$ by subtracting $0.3 \mathrm{dex}$ (as described in their paper). In a recent spectroscopic study of a large sample of globular clusters in M31, Perrett et al. (2002) confirm earlier work that the abundance distribution is bimodal. These authors fit Gaussians with $[\mathrm{Fe} / \mathrm{H}]=$ $-1.44\left(\sigma^{2}=0.22\right)$ and $[\mathrm{Fe} / \mathrm{H}]=-0.5\left(\sigma^{2}=0.13\right)$ to their observations. The solid line in Figure 2 shows the MDF predicted by the new model assuming the sum of contributions from a $\log Z_{c} / Z_{\odot}=-0.55(\sigma=0.2)$ component and from a $\log Z_{c} / Z_{\odot}=-1.5(\sigma=0.3)$ component in the initial ratio of 2.4:1 and normalized to the same area as the observations. The fit is reasonable while possessing the feature of unifying the clusters and stars of each 
population. Note the offset in the peak of the stellar distribution from the assumed value of $\log Z_{c}$ of the metal-rich component.

The simple phenomenological picture outlined above now allows us to model early galaxy evolution based on the near universal appearance of two groups of globular clusters. However, in order to use the model to determine galaxy evolution as a function of time and/or redshift and to calculate star formation rates, we need a relationship between metal abundance and age.

\section{The Age-Metallicity Relation for the Globular Clusters}

In order to determine the age-metallicity relation (AMR) for globular clusters, we refer to the recent papers by VandenBerg (2000) and Salaris \& Weiss (2002). The two studies were combined in the following manner. A zero point of 13.5 Gyr was set for the cluster M92 based on the work of Vandenberg et al. (2002). This required reducing all the ages in the VandenBerg (2000) sample by 0.5 Gyr. A comparison with clusters in common with the Salaris \& Weiss sample was then made and 0.6 Gyr was added to each cluster in this work. Wherever the two studies had clusters in common the VandenBerg ages were used. It will be seen later that imposing this zero point on cluster ages allows only a very narrow range of consistent values of the Hubble constant. These data are shown plotted in Figure 3 against new determinations of $[\mathrm{Fe} / \mathrm{H}]$ by Kraft \& Ivans (2003a, 2003b). The importance of this new globular cluster $[\mathrm{Fe} / \mathrm{H}]$ scale is that the iron abundances were determined from FeII lines rather than FeI and arguably should be more reliable. The uncertainties in the cluster ages are large, but the scatter appears to increase at $[\mathrm{Fe} / \mathrm{H}] \geq-1.6$. In order to account for this apparent increase in scatter in the simplest way, a bifurcated relation was adopted. The form of the relations assumed are

$$
Z / Z_{\odot}=\phi\left(x / x_{s}\right)^{-\alpha} \exp \left(-\left(x / x_{s}\right)^{\beta}\right)
$$

where $x=10^{t_{9}}$ and $t_{9}$ is the lookback time in billions of years. The red cluster relation which extends to the highest $[\mathrm{Fe} / \mathrm{H}]$ has parameters

$$
\phi=0.065 \quad x_{s}=10^{13.08} \quad \alpha=0.15 \quad \beta=1.0
$$

The blue cluster relation follows the above for $[\mathrm{Fe} / \mathrm{H}]<-2.19$, but for larger $[\mathrm{Fe} / \mathrm{H}]$ the parameters are:

$$
\phi=0.0195 \quad x_{s}=10^{13.40} \quad \alpha=0.15 \quad \beta=2.0
$$

These relations are shown superimposed on the data in Figure 3. 
Later an age-metallicity relation will be required for modelling the bulge star formation rate. It is constrained by the observations of Zoccali et al. (2003) showing there are no bulge stars with ages $<10^{10}$ yrs. The relation adopted is shown as the dashed line in Figure 3. Its parameters (valid for $[\mathrm{Fe} / \mathrm{H}]>-1.6$ ) are:

$$
\phi=0.065 \quad x_{s}=10^{13.08} \quad \alpha=0.60 \quad \beta=1.0
$$

\subsection{Calculating the Star Formation Rate}

Equation (5) allows calculation of $\mathrm{d} M_{s} / \mathrm{d} \log \mathrm{Z}$, while equation (11) allows calculation of $\operatorname{dlog} \mathrm{Z} / \mathrm{dt}$. The product of these two quantities then yields the star formation rate $\mathrm{d} M_{s} / \mathrm{dt}$. From (11)

$$
\frac{d \log Z}{d t_{9}}=-\alpha-\beta\left(x / x_{s}\right)^{\beta}
$$

Since the derivation of equations (5)-(10) implicitly assumed instantaneous recycling and $M_{s}$ represents the long lived stars and remnants, the actual SFR will be

$$
\frac{d S}{d t}=\frac{1}{(1-R)} \frac{d M_{s}}{d \log Z}\left(-\frac{d \log Z}{d t_{9}}\right),
$$

where $\mathrm{S}(\mathrm{t})$ is the mass of all stars formed up to time $\mathrm{t}, \mathrm{R}$ is the return fraction (Pagel 1997, p.213) and $\mathrm{t}$ is the evolution time in billions of years. We have assumed $(1-\mathrm{R})=0.7$, appropriate for a Salpeter IMF (Madau et al. 1998). Note that the units of (16) are $\mathrm{M}_{\odot} / \mathrm{yr}$, if the unit of $\mathrm{M}_{t}$ in equations (5)-(10) is $10^{9} \mathrm{M}_{\odot}$.

\subsection{Specifying the Cosmology: The Lookback Time-Redshift Relation}

In order to relate the lookback time and hence $[\mathrm{Fe} / \mathrm{H}]$ via equation (11) to redshift $\mathrm{z}$, we need to specify a cosmological model. The model chosen has $\Omega_{m}=0.3$ and $\Omega_{\Lambda}=\left(1-\Omega_{m}\right)$. The general relation between time and redshift can be found in Peebles (1993, equation 13.20). Letting the Hubble constant be $\mathrm{H}_{0}=100 h \mathrm{~km} \mathrm{~s}^{-1} \mathrm{Mpc}^{-1}$, for the above parameters the age of the universe is $9.426 / \mathrm{h}$ and

$$
\begin{gathered}
z=\left[0.6547 \sinh \left(1.201\left(1-\frac{t_{9} h}{9.426}\right)\right)\right]^{-\frac{2}{3}}-1 \\
t_{9}=\frac{9.426}{h}\left[1-0.8265 \ln \left(1.5275(1+z)^{-\frac{3}{2}}+\left(2.333(1+z)^{-3}+1\right)^{\frac{1}{2}}\right)\right]
\end{gathered}
$$

The unit of the age and lookback time, $t_{9}$, is $10^{9}$ years. 


\section{The Galaxy Formation Scenario}

The goal of this investigation is to re-construct the Madau plot from local observations. This is done by using the above model to chemically evolve a representative co-moving volume of the universe to form what will be a representative or composite galaxy. It may be more than fortuitous that Baldry et al. (2002) showed that the averaged spectra of 2DF galaxies as a function of redshift resemble what would be a present day Sb-Sbc galaxy (i.e. similar to M31 and the Milky Way, galaxies on which our model has been 'calibrated').

\subsection{The Spheroidal Component}

The starting point is to define a representative co-moving volume of the universe which we take to be $100 \mathrm{Mpc}^{3}$. The volume assumed is arbitrary and has no effect on the final result. However, we note that $\phi^{*}$ of a generic galaxy luminosty function is $\sim 0.01 M p c^{-3}$, implying a volume per $L^{*}$ galaxy of $100 M p c^{3}$. Given $\Omega_{b} h^{2}=0.0224$ from WMAP (Spergel et al. 2003), this volume encloses a baryonic mass of $6.22 \times 10^{11} \mathrm{M}_{\odot}$. While as yet we have no way of knowing the mass in remnants left from a Population III phase, it is assumed that within this volume there is at least $1.2 \times 10^{11} \mathrm{M}_{\odot}$ of gas which has been enriched during this earlier phase to $[\mathrm{Fe} / \mathrm{H}]=-4.0$. These numbers make up the $\mathrm{M}_{t}=\left(\mathrm{M}_{t}(\mathrm{blue})+\mathrm{M}_{t}(\mathrm{red})\right)$ and $\mathrm{Z}_{0}$ of our model. To form what will be the main spheroidal component, we assign the parameters shown in Table 1 . As can be seen from the fourth column, most of the parameters are observationally constrained. The cluster parameters, $\log Z_{c} / Z_{\odot}$ and $\sigma$, come from Cote's discussion, and the ratio of $\mathrm{M}_{t}$ (blue) to $\mathrm{M}_{t}(\mathrm{red})$ is the same value deduced from the discussion in $\S 2.3$ of the M31 halo from observations by Durrell et al. The adopted

value of the yield, $\mathrm{p}=0.013\left(\log \left(\mathrm{p} / \mathrm{Z}_{\odot}\right)=-0.11\right)$, is determined from a study of Galactic bulge stars (Zoccali et al. 2003) which will be discussed in more detail in the next section. It should be noted that observational evidence suggests that field halo, cluster stars, thick disk, and bulge stars all exhibit an enhanced oxygen to iron abundance ratio (e.g. Prochaska et al. 2000). This provides the justification for modelling the evolution of the iron abundance under the instantaneous recycling approximation.

Using the parameters given above and equation (16) to calculate the SFR, the results are shown in Figure 4. The final products of the chemical evolution phase are for the blue component $\mathrm{M}_{s}=0.719, \mathrm{M}_{m l}=13.1$, and $\mathrm{M}_{W H I M}=21.2$ and for the red component $\mathrm{M}_{s}=19.6, \mathrm{M}_{m l}=31.7$, and $\mathrm{M}_{W H I M}=33.6$, all in units of $10^{9} \mathrm{M}_{\odot}$. We note from Figure 4 that whereas the SFR of the blue component has ended by $t_{9} \sim 13$ Gyr, the model predicts that the red spheroidal component is still forming stars at $t_{9} \sim 6-7$, remarkably consistent with recent observations of M31 halo stars by Brown et al. (2003). In a later 
section these results will be discussed within the context of a 'toy' model for galaxy formation. Anticipating the main results of this discussion, both $\mathrm{M}_{s, b l u e}$ and $\mathrm{M}_{s, \text { red }}$ will populate the outer halo, $\mathrm{M}_{m l, b l u e}$ will become the bulge, $\mathrm{M}_{m l, r e d}$ will form the disk, while both components of $\mathrm{M}_{W H I M}$ are assumed to be lost from the galaxy.

\subsection{The Bulge Component}

Although the bulge is usually considered to be part of the spheroidal component of a galaxy, it will be discussed separately here. Recently Zoccali et al. (2003) have determined the metallicity distribution of a sample of Galactic bulge stars and find that it resembles the distribution resulting from closed box chemical evolution (equation (1) \& (2) with c=0) with $\log \mathrm{Z}_{0} / \mathrm{Z}_{\odot} \sim-1.6$ and $\log \mathrm{p} / \mathrm{Z}_{\odot} \sim-0.11$. (The mean metallicity of $\mathrm{M}_{m l, b l u e}$ is predicted to be similar to this value of $Z_{0}$ while the the yield implied is the same as that assumed in the model). Further, the above authors also point out that there are no stars with ages $<10^{10}$ yrs in their bulge sample. In order to calculate the SFR associated with this metallicity distribution, the metal-rich component of the adopted age metallicity relation (equations (11) \& (14)) and equation (16) were evaluated with the closed box assumption (equation (1) $\&(2)$ with $\mathrm{c}=0$ ) for the $\mathrm{dM}_{s} / \mathrm{d} \log \mathrm{Z}$ derivative. The results are shown in Figure 4 . The peak in the SFR occurs at $t_{9} \sim 11.3$ and becomes negligble at $t_{9}<10$, in accord with the Zoccali et al. results.

\subsection{The Disk Component}

Just, Fuchs \& Wielen (1996) have developed a method for determining the local disk SFR by modelling the vertical structure of the Galactic disk. Their best model shows a sharp initial maximum followed by a slow decline (Just 2002). In addition, recent infrared studies of the populations in the central parts of the Galaxy (e.g. van Loon et al. 2003), show a very active star formation history. These authors find that the inner Galaxy is dominated by an old population $\left(\geq 7 \times 10^{9} \mathrm{yrs}\right)$ plus an intermediate age population $\left(2 \times 10^{8}-7 \times 10^{9} \mathrm{yrs}\right)$ and even younger stars. These results have prompted the construction of a two component model to represent the disk SFR. The first is a constant SFR of $1 \mathrm{M}_{\odot} / \mathrm{yr}$ for $10^{10} \mathrm{yrs}$ with a Gaussian tail with $\sigma=0.6$. For $t_{9} \leq 10$, the second component is given by

$$
\frac{d S}{d t}=1.5 \times 10^{0.45 z\left(t_{9}\right)}
$$

while for $t_{9}>10$ a Gaussian tail with $\sigma_{t_{9}}=0.6$ was assumed. The sum of these two components is shown in Figure 4. Integrating the rate over time and multiplying the result 
Table 1. Model Parameters

\begin{tabular}{|c|c|c|c|}
\hline Model/equations & Parameter & Value & Source \\
\hline $5-10$ & $\log \left(\mathrm{Z}_{0} / \mathrm{Z}_{\odot}\right)_{\text {blue,red }}$ & $-4.0 \pm 1.0$ & assumed \\
\hline "l & $\mathrm{M}_{t}=\left(\mathrm{M}_{t, \text { blue }}+\mathrm{M}_{t, \text { red }}\right)$ & $1.2 \pm 0.24 \times 10^{11} \mathrm{M}_{\odot}$ & High z observations in Fig. 5 \\
\hline$\prime \prime$ & $\mathrm{M}_{t, \text { red }} / \mathrm{M}_{t, \text { blue }}$ & $2.43 \pm 0.10$ & Durrell et al. (2001) \& $\S 2.3$ \\
\hline$\prime \prime$ & $\log \left(\mathrm{Z}_{c} / \mathrm{Z}_{\odot}\right)_{\text {blue }}$ & $-1.6 \pm 0.1$ & Cote $(1999)$ \\
\hline "I & $\sigma_{\text {blue }}$ & $0.3 \pm 0.1$ & Cote (1999) \\
\hline "I & $\log \left(\mathrm{p} / \mathrm{Z}_{\odot}\right)_{\text {blue }}$ & $-0.11 \pm 0.03$ & Zocalli et al. (2003) \\
\hline$\prime \prime$ & $\mathrm{M}_{W H I M, b l u e}$ & $2.1 \pm 0.42 \times 10^{10} \mathrm{M}_{\odot}$ & from model \\
\hline$\prime \prime$ & $\mathrm{M}_{s, \text { blue }}$ & $0.072 \pm 0,014 \times 10^{10} \mathrm{M}_{\odot}$ & from model \\
\hline$\prime \prime$ & $\log \left(\mathrm{Z}_{c} / \mathrm{Z}_{\odot}\right)_{\text {red }}$ & $-0.55 \pm 0.1$ & Cote (1999) \\
\hline$\prime \prime$ & $\sigma_{\text {red }}$ & $0.3 \pm 0.1$ & Cote (1999) \\
\hline$\prime \prime$ & $\log \left(\mathrm{p} / \mathrm{Z}_{\odot}\right)_{\text {red }}$ & $-0.11 \pm 0.03$ & Zocalli et al. (2003) \\
\hline$\prime \prime$ & $\mathrm{M}_{W H I M, r e d}$ & $3.4 \pm 0.67 \times 10^{10} \mathrm{M}_{\odot}$ & from model \\
\hline$\prime \prime$ & $\mathrm{M}_{s, \text { red }}$ & $1.96 \pm 0.39 \times 10^{10} \mathrm{M}_{\odot}$ & from model \\
\hline $1-3$ & $\mathrm{M}_{t, \text { bulge }}$ & $1.3 \pm 0.26 \times 10^{10} \mathrm{M}_{\odot}$ & $\mathrm{M}_{m l, b l u e}$ from model \\
\hline$\prime \prime$ & $c_{b u l g e}$ & 0 & Zocalli et al. (2003) \\
\hline "I & $\log \left(\mathrm{p} / \mathrm{Z}_{\odot}\right)_{\text {bulge }}$ & $-0.11 \pm 0.03$ & Zocalli et al. (2003) \\
\hline 19 & $\mathrm{M}_{t, d i s k}$ & $3.2 \pm 0.62 \times 10^{10} \mathrm{M}_{\odot}$ & $\mathrm{M}_{m l, \text { red }}$ and $\S 4.3$ \\
\hline \multirow[t]{2}{*}{$17-18$} & Cosmology $\left(\Omega_{m}, \Omega_{\Lambda}, \mathrm{h}\right)$ & $0.3,0.7,0.66$ & assumed \\
\hline & $\mathrm{Z}_{\odot}$ & 0.017 & \\
\hline
\end{tabular}


by the assumed value of $(1-\mathrm{R})=0.7$ gives a model equivalent $\mathrm{M}_{s}$ of $3.2 \times 10^{10} \mathrm{M}_{\odot}$, which is identical with $\mathrm{M}_{m l, r e d}$ (Table 1) and hence consistent with our model. We estimate that the ratio of present SFR to average SFR for this disk component is $\sim 0.6$, which is well within the range expected for an Sb-Sbc galaxy, according to Table 8.2 of Binney \& Merrifield (1998) which is based on data from Kennicutt et al. (1994).

We note from Figure 4 that there is a relatively large overlap in SFR between the disk and the red spheroidal component. Star formation associated with the latter component goes on until $t_{9} \sim 6-7$ Gyr ( $\$ 4.1$ ), implying that the last protogalactic clumps to arrive must have originated at a great distance from the galactic center. This late arrival would allow time for dynamical friction to cause the still quiescently evolving clumps to spiral in to the center of the forming galaxy before colliding to make metal rich globular clusters and to provide gas for the observed ongoing star formation towards the inner galaxy. To observers looking towards the Galactic center, this relatively young, relatively metal rich, relatively high angular momentum 'disk' component could easily appear to be associated with the bulge. One could then understand why the inner metal rich clusters $\left(\mathrm{R}_{g}<4 \mathrm{kpc}\right)$ appear to be associated with the bulge even though they possess significant rotation while the outer red clusters exhibit disk characteristics (Cote 1999, Forbes et al. 2001).

Further, from equation (10), the ratio of mass in red clusters to blue clusters should be equal to the ratio $\mathrm{M}_{t, \text { red }} / \mathrm{M}_{t, b l u e}$ which from Table 1 is $\sim 2.4$. As the observed red to blue cluster ratio is closer to unity, we must assume that either many of the red clusters which do form are disrupted or that the efficiency of red cluster formation is lower due perhaps to tidal effects and to the presence of a substantial existing disk component. In a later discussion, we will attribute the surviving red clusters to a thick disk component. Also part of the thick disk are the stars from disrupted clusters and the earliest formed disk stars resulting from the compression of in situ disk gas from late collisions with still intact protogalactic clumps (assumed to be responsible for the initial burst in the disk SFR at 10 Gyr).

\subsection{The Warm Hot Intergalactic Medium Contribution}

As the model is currently defined, $\mathrm{M}_{W H I M}$ represents the mass loss associated with star formation. The mean metallicity of each component $\left(\mathrm{M}_{W H I M \text {,blue }}\right.$ and $\left.\mathrm{M}_{W H I M, r e d}\right)$ will be the same as the respective stellar components. Assuming that these WHIM components are mixed, we find a total mass of $5.5 \pm 0.80 \times 10^{10} \mathrm{M}_{\odot}$ with a mean $[\mathrm{Fe} / \mathrm{H}]$ of $\sim-1$. As little is presently known concerning the hot gas surrounding an individual galaxy, we refer to the situation in small groups of galaxies. Even here, the metal abundance of hot gas in small groups of galaxies is poorly known, but the lowest determined values are consistent with the 
above mean (Mulchaey 2000). The ratio of the mass in hot gas (the WHIM) to stellar mass is $\sim 0.9 \pm 0.2$, a value similar to what may be only a lower limit deduced from x-ray observations of hot gas in groups of galaxies (Mulchaey 2000). Another statistic discussed by Mulchaey, is the ratio of baryonic mass to total mass which can be evaluated as $\rho\left(M_{t}\right) /\left(\Omega_{m} \rho_{\text {crit }}\right)$. Our model gives $\sim 0.04 \pm 0.01$, which is also the lower end of the distribution found for small groups. Finally, we determine $\Omega_{W H I M}=0.0048 \pm 0.0007$ which compares favorably with the value of $\gtrsim 0.0046 h_{0.66}^{-1}$ from Tripp et al. (2000).

\subsection{The History of the Star Formation Rate Density from Local Observations}

A Madau plot is now easily constructed by adding the SFRs shown in Figure 4 together, dividing by the co-moving volume, taking the logarithm, and plotting the result against the corresponding redshift $\mathrm{z}\left(\mathrm{t}_{9}\right)$. This is shown in Figure 5 along with the data obtained from the high redshift observations. The sources for the observational data, which have been adjusted to our assumed cosmological model and corrected for absorption, are given in the figure caption. Although the model is made up of only four components, there are many parameters involved, and the model is certainly not unique. In theory, there are only two free parameters: $\mathrm{M}_{t}$ and $\log \mathrm{Z}_{0}$. Varying $\log \mathrm{Z}_{0}$ by \pm 1.0 does not change the plot, while a $20 \%$ change in $\mathrm{M}_{t}$ results in $\sim 20 \%$ change in overall normalization. In addition, the figure is insensitive to changes of \pm 0.1 in each of $\log \mathrm{Z}_{c, \text { blue }}, \log \mathrm{Z}_{c \text {,red }}, \sigma_{\text {blue }}, \sigma_{\text {red }}$, and the ratio $\mathrm{M}_{t, \text { red }} / \mathrm{M}_{\text {blue }}$. Varying the yield, $\mathrm{p}$, by \pm 0.03 results in $\sim \mp 23 \%$ changes in overall normalization. The shape of Figure 5 is dependent on the derivatives of the age metallicity relations adopted (equation (15)), and they become very large and rapidly changing at low $[\mathrm{Fe} / \mathrm{H}]$. Finally, the slope of the high redshift tail of Figure 5 is dependent on the adopted value of $\mathrm{h}$ for a given globular cluster age zero point. The masses of the various components obtained from our successful reconstruction of the Madau plot should be relatively robust and allow additional consistency checks on the plausibility of the model. An uncertainty of $\pm 20 \%$ in the value of $\mathrm{M}_{t}$ is assumed and is propagated throughout.

From the model we find $\Omega_{\star}=0.0056 \pm 0.0007$, which can be compared with the Fukugita, Hogan \& Peebles $(1998, \mathrm{FHP})$ value of $\Omega_{\star}=0.0037_{-0.0013}^{+0.0019} \mathrm{~h}_{0.66}^{-1}$. Although the error bars overlap, our value is $\sim 50 \%$ higher. Further, the model shows that $\mathrm{M}_{\text {disk }} / \mathrm{M}_{\text {spheroid }}=0.9 \pm 0.2$ compared to $\sim 0.3$ from FHP, $\sim 1$ from Schechter \& Dressler (1987), and $\sim 1.3 \pm 0.2$ from Benson et al. ( 2002). Finally, the gas from the Population III phase that did not go into making the galaxy is considered to be a diffuse component and contributes $\Omega_{\text {diffuse }}=$ $0.044 \pm 0.002$. (This assumes that the mass in dark condensed remnants is negligible). Here it is considered separate from the WHIM and tentatively identified with the gas responsible 
for producing the local Lyman $\alpha$ forest which apparently could significantly exceed $32 \%$ of all local baryons (Stocke et al. 2003).

Two additional consistency checks on our model are now considered. The first is concerned with the origin of the damped Lyman $\alpha$ systems (DLA's) for which any complete model of chemical evolution applied to the entire universe should account. Figure 6 shows the assumed age-metallicity relations (converted to metallicity-redshift relations) on which are superimposed a selection of observations of DLA's from Kulkarni \& Fall (2002) and Prochaska et al. (2003). Recall that the two relations apply to each of the metal poor clusters, and metal rich clusters. In order to make a robust statistical estimate of the probability that any particular component is intersected by a random line of sight, one needs to know the distribution in area as well as the number and size of the protogalactic clumps, parameters which are not specified by the model. At most, we can calculate the amount of gas remaining in each component as a function of $[\mathrm{Fe} / \mathrm{H}]$. The range over which this amount is $>1 \%$ of the original amounts of 3.5 , and $8.5 \times 10^{10} \mathrm{M} \odot$ is indicated by the thickened parts of the lines in Figure 6. As it stands the model does not predict either an age-metallicity relation nor a metallicity distribution function for the disk component, but only the mass of gas that is available, $3.2 \times 10^{10} \mathrm{M}_{\odot}$. This comparison with the observations of DLA's is necessarily crude, but there appear to be no major inconsistencies.

Secondly, we note that both the large reservoir of low angular momentum gas released after the first collapse and a conduit to the center of the galaxy (along the rotation axis) are ingredients required for fuelling and possibly creating a black hole. Thus, the model naturally provides an environment conducive to the establishment of a relationship between the mass of a central black hole and of the surrounding bulge stars. If $\mathrm{M} \bullet / \mathrm{M}_{\text {bulge }}=0.002$ (Magorrian et al. 1998), then the mass of the black hole in the model is $\mathrm{M} \bullet=2.6 \pm 0.5 \times 10^{7} \mathrm{M}_{\odot}$, comparable to the black hole mass in the nucleus of M31. When divided by the assumed comoving volume one finds $\rho_{\bullet}=2.6 \pm 0.5 \times 10^{5} \mathrm{M}_{\odot} / \mathrm{Mpc}^{3}$ for the local black hole density which is in excellent agreement with the recent determination of this quantity by $\mathrm{Yu} \&$ Tremaine (2002). Further, the maximum SFR of the bulge component occurs at redshift $\mathrm{z} \sim 2.3$, which is close to the redshift at which the space density of luminous quasars is a maximum.

\subsection{Colliding Boxes and Galaxy Formation}

The main quantitative results of this investigation are contained in Figure 5 and Table 3. What follows is a proposed physical framework which could accommodate the chemical evolution scenario described above. Basically, we envision the anisotropic collapse of a num- 
ber of initially isolated, quiescently evolving, protogalactic clumps. The initial configuration is then either a triaxial halo (CDM) or a fragmented sheet or flattened filament (WDM). Evidence which supports these initial conditions is the triaxial spatial distribution of the outlying satellites of the Galaxy (Hartwick 2000) along with a similar alignment of the space distribution and space motions of the blue globular clusters (Hartwick 2002). During the first collapse, which is transverse to the long (rotation) axis, the low angular momentum clumps will collide to form the blue globular clusters. In turn, the gas released in the collisions will fall towards the center to form the bulge. Support for a low angular momentum halo-bulge connection can be found in the review of Wyse et al. (1997), who show (their Figure 7) that the distribution of the specific angular momentum of the (classical) halo and bulge are similar and unlike the disk/thick disk distribution. The higher angular momentum clumps will generally not collide initially, but instead will fall towards the center along the long axis while still forming stars and enriching the gas within them. These clumps will eventually meet at what will become the plane of the disk. Red clusters then form in these collisions, and the gas released settles down to form the disk. The stars which had previously formed continue their dynamical evolution back out into the halo. The MDF of this group of stars should be similar to the dashed curve in Figure 2, with a peak at $[\mathrm{Fe} / \mathrm{H}] \sim-0.8$ and with an angular momentum distribution like the thin disk. Note that there is a metal poor tail associated with this MDF. The existence of a relatively high angular momentum (disk-like), relatively metal rich, and relatively massive component of the Galactic halo is an inescapable (and falsifiable) prediction of this model.

In $\S 4.3$ we noted that according to our model there should be $\sim 2$ times more red clusters than blue being formed. However, given the fact that the SFR of the disk and red component overlap (Figure 4), the later infalling clumps are more likely to collide with existing disk gas rather than other clumps or be tidally disrupted. It is assumed that not only will many fewer red clusters be formed, but that the compression of this gas from infalling clumps will form stars whose kinematics can be expected to resemble the red clusters which survive in numbers comparable to blue clusters. (In equation (10) $\eta$ is effectively reduced by $\sim 1 / 2$ ). A thick disk component is thus created. The peak in the disk SFR at 10 Gyr in Figure 4 is identified with this process, and the mass in the thick disk was estimated by integrating over this peak to $t_{9} \sim 9.1$. The result is $\mathrm{M}_{T D}=1.04 \times 10^{10} \mathrm{M}_{\odot}$.

A further consequence of the overlap in SFR referred to above is that there will also be an overlap both in age and metal abundance among metal poor thick disk stars and metal rich red halo stars. An important difference is that the thick disk as defined above will not possess a metal poor tail unlike the red halo stars. In addition to the metal rich clusters, the thick disk could also be made up of the first stars to form from the gas of the $\mathrm{M}_{m l, r e d}$ component with mean $\langle[\mathrm{Fe} / \mathrm{H}]\rangle \sim-0.7, \sigma \sim 0.3$ (i.e. there should be relatively 
few stars with $[\mathrm{Fe} / \mathrm{H}] \leq-1$ ) so that at a minimum the MDF might resemble that of the metal rich clusters $(\langle[\mathrm{Fe} / \mathrm{H}]\rangle \sim-0.55, \sigma \sim 0.3)$. The disk component is not chemically (nor dynamically) evolved here so it is not possible to predict just how kinematically and chemically distinct will be the stellar component formed in the spike of the disk SFR at 10 Gyr in Figure 4 which we consider to be part of the thick disk (but see the discussion below for a possible Milky Way counterpart).

Except for one possible caveat (dynamical evolutionary effects such as dynamical friction for example) the vertical scale height of the blue halo component (including the blue globular clusters) should be comparable to the red halo component so that the metal poor blue globular clusters and the metal rich red halo field stars will occupy the galaxy halo contemporaneously. This result could provide a natural explanation for the observations of Forte et al. (1981) who showed that the integrated light from halo stars was redder than that of the globular clusters at the same projected radius in four galaxies in the Virgo cluster. Subsequently, similar results have been found in other galaxies including the Milky Way and M31. The effect is attributed to a difference in metal abundance between the two populations (e.g. Harris, 1991) in agreement with the prediction. Below we will argue that the above caveat applies to the Milky Way with the result that the red component is more flattened than the blue.

Table 2 summarizes the relative sizes of the various components of our framework galaxy. More sophisticated modelling is required to turn the mass ratios in Table 2 into local density ratios. It should also be emphasized that this is a composite model based on observations of the Galaxy and M31 although it likely more closely resembles M31. While we expect all of its components to be present in both galaxies, the actual ratios will undoubtedly be different.

We now consider whether the predicted red halo component and the thick disk as defined by the framework model actually do have counterparts in the Milky Way. A high latitude deep CCD survey has recently been discussed by Karaali et al. (2003) (see also Chen et al. 2001). By using ultraviolet excess as a metallicity indicator they show (their Fig. 11) the distribution of stars as a function of metallicity in progressively fainter magnitude bins. The diagrams show 3 peaks at $[\mathrm{Fe} / \mathrm{H}] \sim-0.06,-0.83$, and -1.59 which the authors attribute to thin disk, thick disk, and halo respectively. Comparing these diagrams with Fig. 4 of Beers (1999) which shows the (admittedly biased) MDF of metal poor star candidates, we see very good correspondence with the two metal poorest distributions. These MDF's are just what our two component halo model predicts, although the red halo component apparently manifests itself as the thick disk component of Fig. 11 above. This identification would allow a natural explanation for the 'metal weak thick disk' stars (e.g. Morrison et al. 1990, Beers et al. 
2002) and the flattened component of the 'classical' halo tracers as represented by the RR Lyrae stars (Hartwick, 1987) and blue horizontal branch stars (Kinman et al. 1994) as the expected metal poor tail of the red halo component. The observed flattening is most likely a result of dynamical evolution and hence is not predicted by our framework model which is chemical evolution based. Binney \& May (1986) have shown that a thick disk structure is expected if the angular momentum of the stars formed in the infalling proto-disk material is not parallel to a principal axis of the early spheroid. For the Milky Way, some evidence for such a misalignment exists (Hartwick, 2000, 2002). Whatever the mechanism, the apparent flattening along with the predicted relatively high angular momentum of the red component, when observed against a background of the blue (classical low angular momentum) stellar halo component, should show a gradient in circular velocity with respect to height above the plane which is qualitatively consistent with that seen in Fig. 4 of Chiba \& Beers (2000). Carney (2001) finds a similar result (his Fig. 3) and concludes that there are at least two populations of metal poor stars one of which is flattened and rotating relatively rapidly.

On the basis of the above discussion, we expect the bulk of the stars of the red halo component to occupy a flattened distribution. However, it would not be surprising if some of the clumps, while being dragged down, became tidally disrupted, resulting in some metal rich stars being found at large distances from the Galactic plane. Ratnatunga and Freeman (1989) showed that at $\sim 5 \mathrm{kpc}$ above the plane the number of $\mathrm{K}$ giants with $[\mathrm{Fe} / \mathrm{H}] \geq-1$ is 12 out of 32. Recently, Morrison et al. (2003) presented preliminary results of a more distant survey for halo stars and find 5 out of 30 stars with $[\mathrm{Fe} / \mathrm{H}] \geq-1$ at distances of order $30 \mathrm{kpc}$.

As emphasized earlier, the model thick disk does not have a metal poor tail and hence is different from the thick disk identified above. In our Galaxy, we identify its 'backbone' with the metal rich globular clusters and with the observed stellar component referred to as a thick disk by Bell (1996) (mean $[\mathrm{Fe} / \mathrm{H}]=-0.36)$, Carney $(2001)$ (mean $[\mathrm{m} / \mathrm{H}] \sim-0.4$ as estimated by this author from his Fig.5a), and Soubiran et al. (2003) (mean $[\mathrm{Fe} / \mathrm{H}]=-0.48$ ) with all these workers determining a rotational lag of $40-50 \mathrm{~km} \mathrm{~s}^{-1}$. This component, which is expected to partially overlap the red halo both in $[\mathrm{Fe} / \mathrm{H}]$ and age, must then be buried in the metal poor tail of the thin disk component of Fig. 11 referred to above. Whether or not two distinct stellar thick disk components exist cannot be resolved here although such a possibility was hinted at in the work of Soubiran et al. (see Norris 1999 for a recent report on the status of the Galactic thick disk).

We conclude that Milky Way counterparts to the components of the framework model do exist. It is interesting that both of the above components may have been anticipated by the participants of the Vatican Conference on Stellar Populations (O'Connell, 1958). 
There, Oort (1958) argued for the existence of an 'intermediate Population II' consisting of stars which formed during the brief contracting stage (possible identification here with our red halo). Likewise Baade (1958) argued for a 'disk Population II' by analogy with such a population in M31 and from the spatial distribution of what are referred to here as the red globular clusters which form the backbone of our thick disk.

\section{Summary and Conclusions}

Starting with a new chemical evolution model and with the goal of accounting for the bimodal distribution of globular clusters, the cosmic star formation rate density history was successfully modelled with a minimum number of free (i.e. non-observationally determined) parameters. The resulting model compares favorably with the Madau plot and thus provides a scenario for the chemical evolution of a representative sample of the universe. The model was obtained by constructing a composite galaxy based on observational input from the Milky Way and M31. An important aspect of this result as presented is that it is independent of the effects of dust extinction. While it might be considered presumptuous to expect to describe the entire universe with a single galaxy, the summary of ancillary quantities listed in Table 3 shows that many local cosmological constraints are also satisfied in varying degrees.

A physical framework which incorporates the above results is presented. The picture involves a large scale anisotropic collapse of quiescently evolving, isolated protogalactic clumps. During the first collapse (transverse to the rotation axis), only the low angular momentum clumps collide resulting in the formation of the blue clusters and a reservoir of low angular momentum gas which later is turned into bulge stars. As a result of the second collapse (along the rotation axis), red clusters are formed which become the backbone of a thick disk component. The stars already formed in the clumps before they collide pass through and back into the halo. An important prediction of the model is that the Galactic halo should contain a relatively metal rich $\left([\mathrm{Fe} / \mathrm{H}]_{\text {peak }} \sim-0.8\right)$ population of stars with disk-like angular momentum. Galactic counterparts of this red halo component and model thick disk are identified and are predicted to partially overlap in metal abundance, age and spatial distribution. This overlap might explain why the properties of the 'thick disk' have been so difficult to define up to now (e.g. Norris, 1999).

The author wishes to thank Drs Ray Carlberg, Chris Pritchet and Sidney van den Bergh

for commenting on earlier versions of this paper. He also wishes to acknowledge financial support from an NSERC (Canada) discovery grant. 
Table 2. A Framework Model

\begin{tabular}{ll}
\hline \multicolumn{1}{c}{ Component } & $\mathrm{M} / \mathrm{M}_{\text {disk }}$ \\
\hline Disk & 1.0 \\
Thick Disk & 0.33 \\
Surviving Red Globular Clusters & 0.000751 \\
Bulge & 0.42 \\
Red Stellar Halo & 0.63 \\
Blue Stellar Halo & 0.023 \\
Blue Globular Clusters & 0.000751 \\
\hline
\end{tabular}

Table 3. Summary of Results

\begin{tabular}{cccc}
\hline \hline Parameter & Model & Observed & Reference \\
\hline$\Omega_{\star}$ & $0.0056 \pm 0.0007$ & $0.0037_{-0.0013}^{+0.0019} h_{0.66}^{-1}$ & FHP $(1998)$ \\
$\Omega_{\text {WHIM }}$ & $0.0048 \pm 0.0007$ & $\gtrsim 0.0046 h_{0.66}^{-1}$ & Tripp et al. $(2000)$ \\
$\Omega_{\text {diffuse }}$ & $0.044 \pm 0.002$ & $\geq 0.0164 h_{0.66}^{-2}$ & Stocke et al. $(2003)$ \\
$\langle[F e / H]\rangle_{W H I M}$ & $\sim-1$ & $\geq-1$ & Mulchaey $(2000)$ \\
$\mathrm{M}_{W H I M} / \mathrm{M}_{\star}$ & $0.9 \pm 0.2$ & $>1 ?$ & Mulchaey $(2000)$ \\
$\mathrm{M}_{\star, \text { disk }} / \mathrm{M}_{\star, \text { spheroid }}$ & $0.9 \pm 0.2$ & $\sim 1$ & Schechter \& Dressler $(1987)$ \\
$\rho_{\left(M_{t}\right) /\left(\Omega_{m} \rho_{\text {crit }}\right)}$ & $0.04 \pm 0.01$ & $\geq 0.04$ & Mulchaey $(2000)$ \\
$\rho_{\bullet}\left(\mathrm{M}_{\odot} / \mathrm{Mpc}^{3}\right)$ & $2.6 \pm 0.5 \times 10^{5}$ & $2.8 \pm 0.4 \times 10^{5} h_{0.66}^{2}$ & Yu \& Tremaine $(2002)$ \\
\hline
\end{tabular}




\section{REFERENCES}

Adelberger, K.L., \& Steidel, C.C. 2000, ApJ, 544, 218

Ashman, K.M., \& Zepf, S.E. 1998, Globular Cluster Systems (Cambridge: Cambridge University Press)

Baade, W. 1958, in Stellar Populations, ed. D.J.K. O'Connell, (Amsterdam: North Holland Publishing Company), p. 303

Baldry, I.K., Glazebrook, K., Baugh, C.M., Bland-Hawthorn, J., Bridges, T.. Cannon, R., Cole, S., et al. 2002, ApJ, 569, 582

Barmby, P., Huchra, J.P., Brodie, J.P., Forbes, D.A., Schroder, L.L., \& Grillmair, C.J. 2000, AJ, 119, 727

Beasley, M.A., Baugh, C.M., Forbes, D.A., Sharples, R.M., \& Frenk, C.S. 2002, MNRAS, 333,383

Becker, W. 1950, Sterne und Sternsysteme, 2nd ed. (Dresden: T. Steinkopff)

Beers, T.C. 1999, in The Third Stromlo Symposium: The Galactic Halo, ed. B.K. Gibson, T.S. Axelrod, \& M.E. Putman, (San Francisco: ASP), 202

Beers, T.C., Drilling, J.S., Rossi, S., Chiba, M., Rhee, J., Fuhrmeister, B., Norris, J.E., \& von Hippel, T. 2002, AJ, 124, 931

Bell, D.J. 1996, PASP, 108, 1139

Benson, A.J., Frenk, C.S., \& Sharples, R.M. 2002, ApJ, 574, 104

Binney, J., \& May, A. 1986, MNRAS, 218, 743

Binney, J., \& Merrifield, M. 1998, Galactic Astronomy, (Princeton: Princeton University Press)

Brown, T.M., Ferguson, H.C., Smith, E., Kimble, R.A., Sweigart, A.V., Renzini, A., Rich, R.M., \& VandenBerg, D.A. 2003, ApJ, 592, L17

Carney, B.W. 2001, in Dynamics of Star Clusters and the Milky Way, ed. S. Deiters, B. Fuchs, A. Just, R. Spurzem, \& R. Wielen, (San Francisco: ASP), 255

Chen, B., Stoughton, C., Smith, J.A., Uomoto, A., Pier, J.R., Yanny, B., Ivezic , Z., et al. 2001, ApJ, 553, 184

Chiba, M., \& Beers, T.C. 2000, AJ, 119, 2843

Cote, P. 1999, AJ, 118, 406

Cote, P., Marzke, R.O., West, M.J., \& Minniti, D. 2000, ApJ, 533, 869

Cote, P., West, M.J., \& Marzke, R.O. 2002, ApJ, 567, 853 
Durrell, P.R., Harris, W.E., \& Pritchet, C.J. 2001, AJ, 121, 2557

Eggen, O.J., Lynden-Bell, D. \& Sandage, A. 1962, ApJ, 136, 748

Flores, H., Hammer, F., Thuan, T.X., Csarsky, C., Desert, F.X., Omont, A., Lilly, S.J., Eales, S., Crampton, D., \& Le Fevre, O. 1999, ApJ, 517, 148

Forbes, D.A., Brodie, J.P., \& Larsen, S.S. 2001, ApJ, 556, L83

Forte, J.C., Strom, S.E., \& Strom, K.M. 1981, ApJ, 245, L9

Fukugita, M., Hogan, C.J., \& Peebles, P.J.E. 1998, ApJ, 503, 518 (FHP)

Gebhardt, K., \& Kissler-Patig, M. 1999, AJ, 118, 1526

Giavalisco, M., Dickinson, M., Ferguson, H.C., Ravindranath, S., Kretchmer, C., Moustakas, L.A., Madau, P., Fall, S.M. et al. 2003, astro-ph/0309065

Goudfrooij, P., Strader, J., Brenneman, L., Kissler-Patig, M., Minniti, D., \& Huizinga, J.E. 2003, MNRAS, 343, 665

Gunn, J.E., 1980, in Globular Clusters, ed. D. Hanes \& B. Madore (Cambridge: Cambridge University Press), 301

Gwyn, S.D.J. 2001, The Evolution of Galaxies in the Hubble Deep Fields, PhD thesis, University of Victoria

Harris, W.E. 1991, Ann Rev Ast \& Ap, 29, 543

Hartwick, F.D.A. 1976, ApJ, 209, 418

Hartwick, F.D.A. 1987, in The Galaxy, ed. G. Gilmore \& R. Carswell (Dordrecht: Reidel), 281

Hartwick, F.D.A. 1999, in IAU Symposium 190, New Views of the Magellanic Clouds, ed. Y.-H. Chu, N.B. Suntzeff, J.E. Hesser, \& D.A. Bohlender, (San Francisco: ASP), 8

Hartwick, F.D.A. 2000, AJ, 119, 2248

Hartwick, F.D.A. 2002, ApJ, 576, L29

Huchra, J.P., Brodie, J.P., \& Kent, S.M. 1991, ApJ, 370, 495

Hughes, D.H., Serjeant, S., Dunlop, J., Rowan-Robinson, M., Blain, A., Mann, R.G., Ivison, R., et al. 1998, Nature, 394, 241

Just, A. 2002, in Disks of Galaxies: Kinematics, Dynamics, and Perturbations, ed. E.Athanassoula, A. Bosma, \& R. Mujica, (San Francisco: ASP), 117

Just, A., Fuchs, B., \& Wielen, R. 1996, A\&A, 309, 715

Karaali, S., Ak, S.G., Bilir, S., Karatas, Y., \& Gilmore, G. 2003, MNRAS, 343 , 1013 
Kennicutt, R.C., Tamblyn, P., \& Congdon, C.W. 1994, ApJ, 435, 22

Kinman, T. D. 1959, MNRAS, 119, 538

Kinman, T.D., Suntzeff, N.B., \& Kraft, R.P. 1994, AJ, 108, 1722

Kraft, R.P., \& Ivans, I.I. 2003a, PASP, 115, 143

Kraft, R.P., \& Ivans, I.I. 2003b, in Origin and Evolution of the Elements, ed. A McWilliam \& M Rauch (Pasadena: Carnegie Observatories)

Kravtsov, A.V., \& Gnedin, O.Y. 2003, astro-ph/0305199

Kulkarni, V.P., \& Fall, S.M. 2002, ApJ, 580, 732

Kundu, A., \& Whitmore, B.C. 2001, AJ, 121, 2950

Lilly, S.J., Le Fevre, O., Hammer, F., \& Crampton, D. 1996, ApJ, 455, L1

Madau, P., Ferguson, H., Dickinson, M., Giavalisco, M., Steidel, C.C. \& Fruchter, A. 1996, MNRAS, 283, 1388

Madau, P., Pozzetti, L., \& Dickinson, M. 1998, ApJ, 498, 106

Magorrian, J., Tremaine, S., Richstone, D., Bender, R., Bower, G., Dressler, A., Faber, S.M., et al. 1998, AJ, 115, 2285

McCrea, W.H. 1982, in Progress in Cosmology, ed A.W. Wolfendale (Dordrecht: Reidel), 239

McLaughlin, D.E. 1999, AJ, 117, 2398

Morgan, W.W. 1959, AJ, 64, 432

Morrison, H.L., Flynn, C., \& Freeman, K.C. 1990, AJ, 100, 1191

Morrison, H.L., Norris, J., Mateo, M., Harding, P., Olszewski, E.W., Shectman, S.A., DohmPalmer, R.C., Helmi, A., \& Freeman, K.C. 2003, AJ, 125, 2502

Mulchaey, J.S. 2000, Ann Rev Ast \& Ap, 38, 289

Norris, J.E. 1999, Ap\&SS, 265, 213

O'Connell, D.J.K., ed. 1958, Stellar Populations, (Amsterdam: North Holland Publishing Company)

Oort, J.H. 1958, in Stellar Populations, ed. D.J.K. O'Connell, (Amsterdam: North Holland Publishing Company), p. 415

Pagel, B.E.J. 1997, Nucleosynthesis and Chemical Evolution of Galaxies, ( Cambridge: Cambridge University Press)

Peebles, P.J.E. 1993, Principles of Physical Cosmology, (Princeton: Princeton University Press) 
Perritt, K.M., Bridges, T.J., Hanes, D.A., Irwin, M.J., Brodie, J.P., Carter, D., Huchra, J.P., \& Watson, F.G. 2002, AJ, 123, 2490

Prochaska, J.X., Gawiser, E., Wolfe, A.M., Castro, S., \& Djorgovski, S.G. 2003, ApJ, 595, L9

Prochaska, J.X., Naumov, S.O., Carney, B.W., McWilliam, A., \& Wolfe, A.M. 2000 , AJ, 120,2513

Ratnatunga, R.U., \& Freeman, K.C. 1989, ApJ, 339, 126

Salaris, M., \& Weiss, A. 2002, A\&A, 388, 492

Schechter, P.L., \& Dressler, A. 1987, AJ, 94, 563

Schweizer, F. 1987, in Nearly Normal Galaxies: From the Planck Time to the Present, ed. S.M. Faber (Berlin: Springer), 18

Searle, L. \& Zinn, R. 1978, ApJ, 225, 357

Somerville, R.S., Primack, J.R., \& Faber, S.M. 2001, MNRAS, 320, 504

Soubiran, C., Bienayme, O., \& Siebert, A. 2003, A\&A, 398, 141

Spergel, D.N., Verde, L., Peiris, H.V., Komatsu, E., Nolta, M.R., Bennett, C.L. , Halpern, M., et al. 2003, astro-ph/0302209

Steidel, C.C., Adelberger, K.L., Giavalisco, M., Dickinson, M., \& Pettini, M. 1999, ApJ, 519, 1

Stocke,J.T., Penton, S.V., \& Shull, J.M. 2003, in The IGM/Galaxy Connection: The Distributions of Baryons at $\mathrm{Z}=0$, ASSL Conference Proceedings Vol. 281., ed. Jessica L. Rosenberg \& Mary E. Putman, (Dordrecht: Kluwer), 57

Treyer, M.A., Ellis, R.S., Milliard, B., Donas, J., \& Bridges, T.J. 1998, MNRAS, 300, 303

Tripp, T.M., Savage, B.D., \& Jenkins, E.B. 2000, ApJ, 534, L1

VandenBerg, D.A. 2000, ApJS, 129, 315

VandenBerg, D.A., Richard, O., Michaud, G., \& Richer, J. 2002, ApJ, 571, 487

van Loon, J.Th., Gilmore, G.F., Omont, A., Blommaert, J.A.D.L., Glass, I.S., Messineo, M., Schuller, F., et al. 2003, MNRAS, 338, 857

Wilson, G., Cowie, L.L., Barger, A.J., \& Burke, D.J. 2002, AJ, 124, 1258

Wyse, R.F.G., Gilmore, G., \& Franx, M. 1997, Ann Rev Ast \& Ap, 35, 637

Yu, Q., \& Tremaine, S. 2002, MNRAS, 335, 965

Zinn, R. 1985, ApJ, 293, 424 
Zoccali, M., Renzini, A., Ortolani, S., Greggio, L., Saviane, I., Cassisi, S., Rejkuba, M., et al. 2003, A\&A, 399, 931 
Fig. 1.- An illustration of the results from the new chemical evolution model assuming a hypothetical group of globular clusters with Gaussian metallicity distribution parameters $\log \mathrm{Z}_{c} / Z_{\odot}=-1.0$ and $\sigma=0.3$. Shown are the cumulative distributions of stars, $\mathrm{M}_{s}$ (solid line), of gas, $\mathrm{M}_{g}$ (dashed line), of recyclable gas lost, $\mathrm{M}_{m l}$ (dot-dashed line), and of mass lost due to star formation, $\mathrm{M}_{W H I M}$ (dotted line). Lowering $\log \mathrm{Z}_{c} / \mathrm{Z}_{\odot}$ leads to a decrease in the ratio of stars formed to gas lost and vice versa.

Fig. 2.- The observed $[\mathrm{Fe} / \mathrm{H}]$-frequency histogram of M31 halo stars from Durrell et al. (2001) on which is superposed the results from the chemical evolution model normalized to the same area. The model parameters are $\log \mathrm{Z}_{c, \text { red }} / Z_{\odot}=-0.55, \sigma_{\text {red }}=0.2, \log \mathrm{Z}_{c, \text { blue }} / \mathrm{Z}_{\odot}=$ $-1.5, \sigma_{\text {blue }}=0.3, \mathrm{M}_{t, \text { red }} / \mathrm{M}_{t, \text { blue }}=2.43, \mathrm{p}=0.013, \log \mathrm{Z}_{0} / \mathrm{Z}_{\odot}=-4.0$. Also shown are the contributions from the blue component (dotted line), the red component (dashed line), and the sum (solid line).

Fig. 3.- The age-metallicity relations for Galactic globular clusters. Open circles-data from VandenBerg (2000), and Salaris \& Weiss (2002) combined as described in the text. The solid line is the adopted relation for blue clusters (equations $11 \& 13$ ), the dot-dashed line is the adopted relation for the red clusters (equations $11 \& 12$ ), and the dashed line is the adopted relation for the bulge component (equations $11 \& 14$ ). A representative error bar is also shown.

Fig. 4. - Star formation rates as a function of lookback time derived for each component. Shown are the blue spheroidal component (dotted line), the red spheroidal component (solid line), the bulge (short dashes), and the disk (long dashes).

Fig. 5.- The Madau plot (star formation rate density versus redshift). The solid line is the the model. The observational data (with error bars) are as follows: Flores et al. (1999) (open triangle), Treyer et al. (1998) (closed pentagon), Wilson et al. (2002) (closed circles), Hughes et al. (1998) (open circle), Giavalisco et al. (2003) (open squares), Steidel et al. (1999) (closed triangles), and Gwyn (2001) (closed diamonds). Adjustment of the data for cosmology and dust extinction follows from Somerville et al. (2001) for the first four data sets and from Adelberger \& Steidel (2000) for the last three.

Fig. 6. - The age-metallicity relations transformed to the $[\mathrm{Fe} / \mathrm{H}]$-redshift plane on which is superposed observational data for damped Lyman $\alpha$ systems. Closed circles-[Zn/H] determinations discussed by Kulkarni \& Fall (2002), closed triangles-HI column density weighted values of $[\mathrm{M} / \mathrm{H}]$ from Prochaska et al. (2003). Both plotted quantities are $[\mathrm{Fe} / \mathrm{H}]$ surrogates which account for depletion onto interstellar grains. The heavy lines indicate the region where the gas remaining is greater than $1 \%$ of its initial value. Solid line-the blue cluster relation, and dot-dashed line-the red cluster relation. 


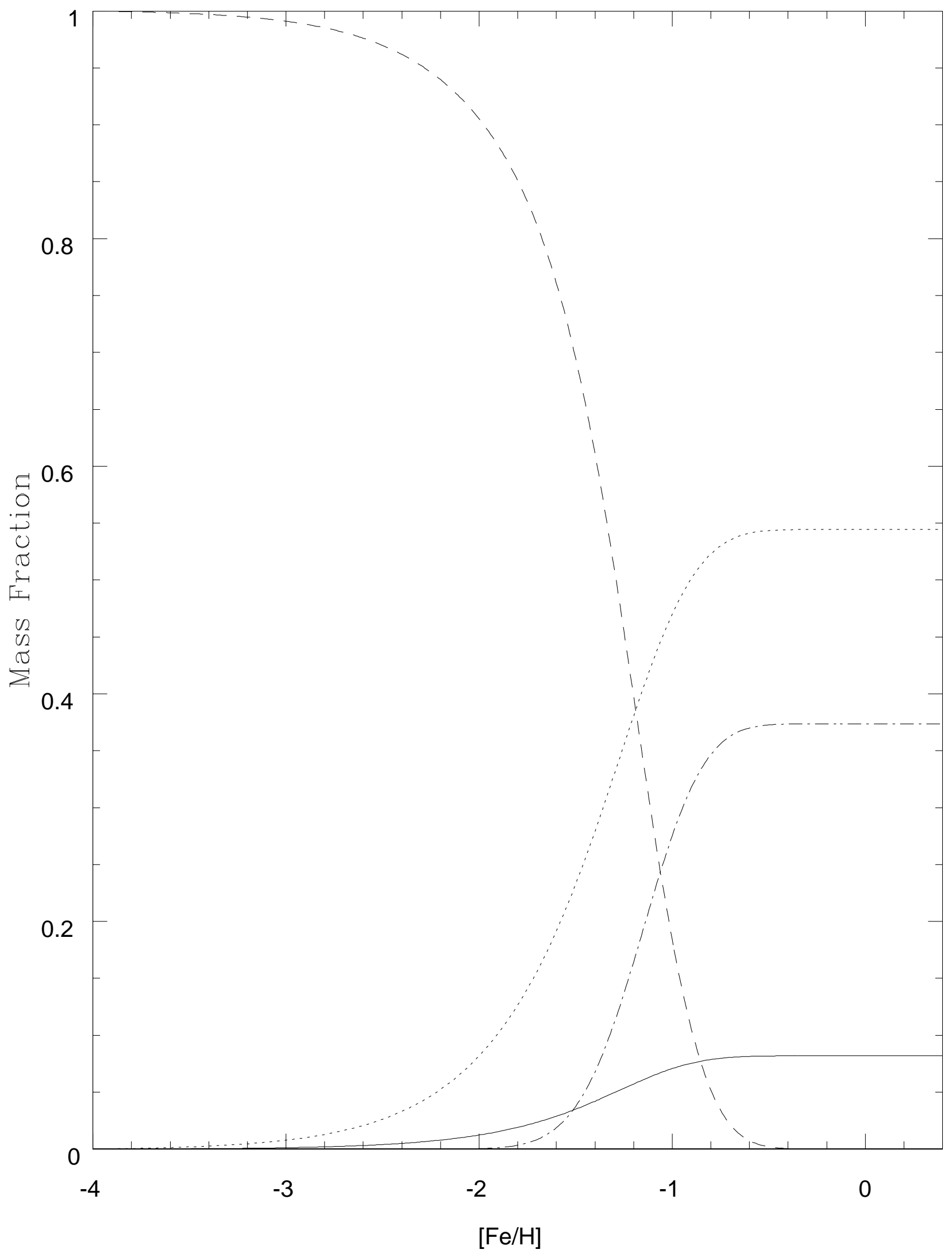




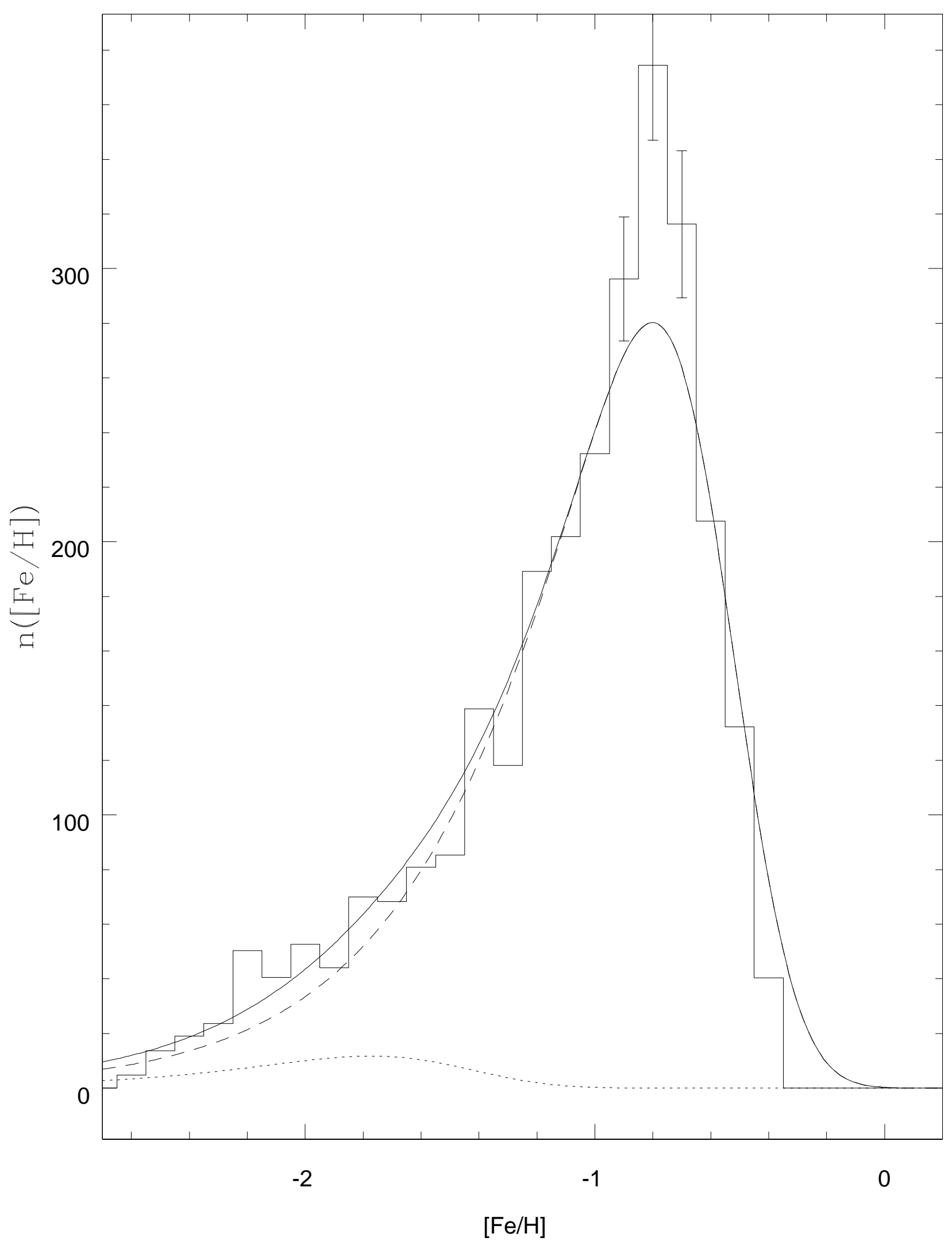




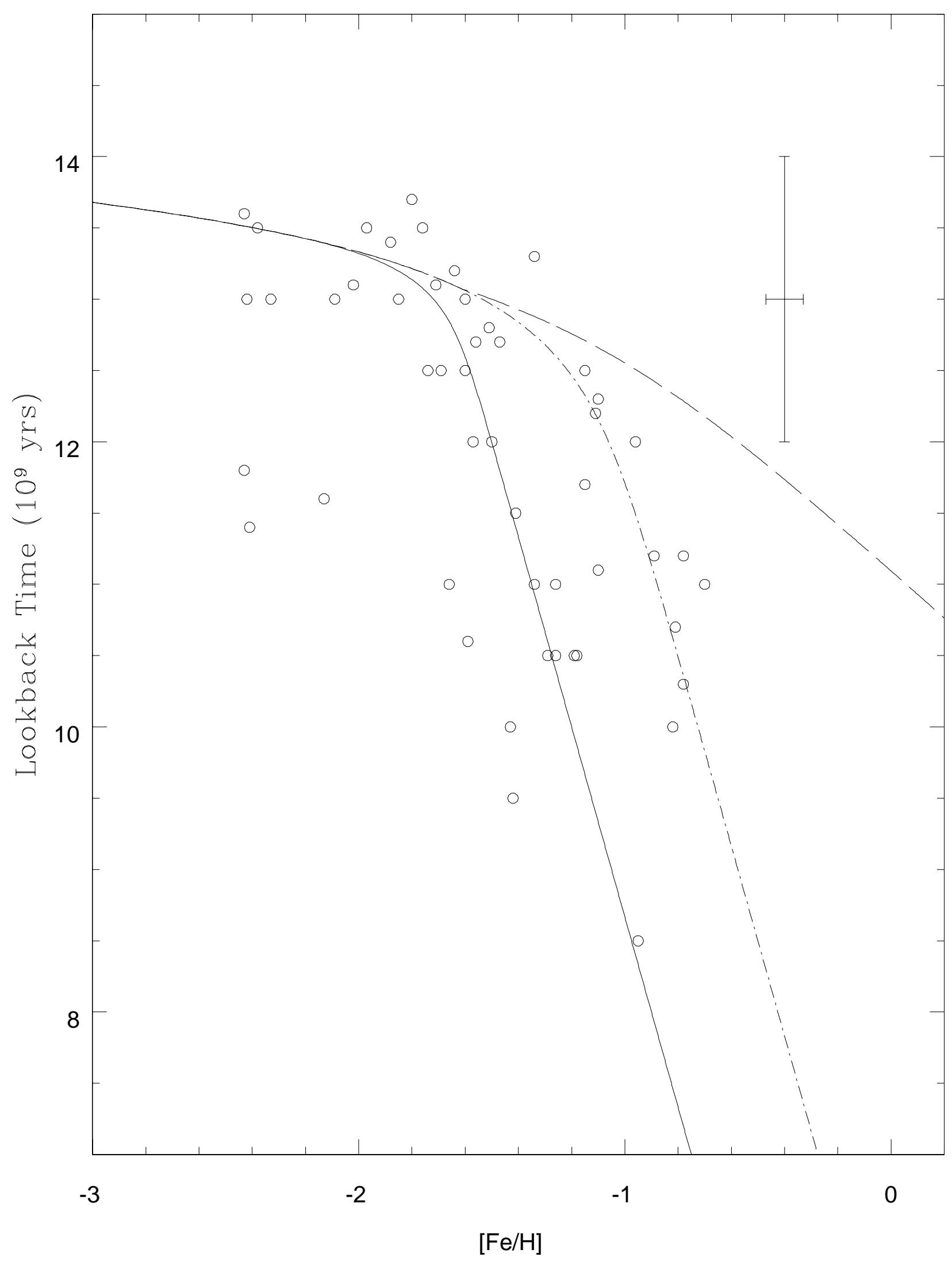




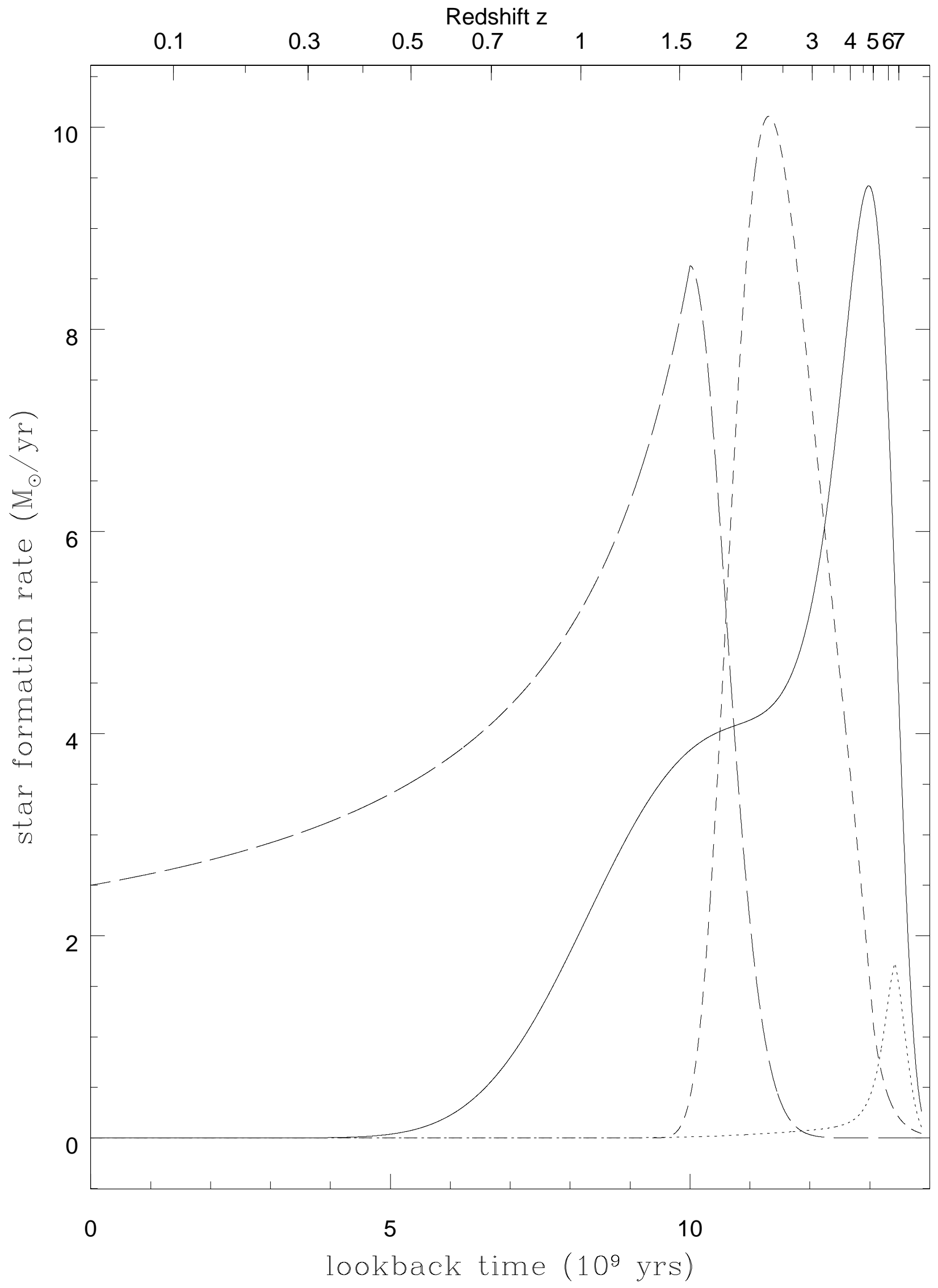




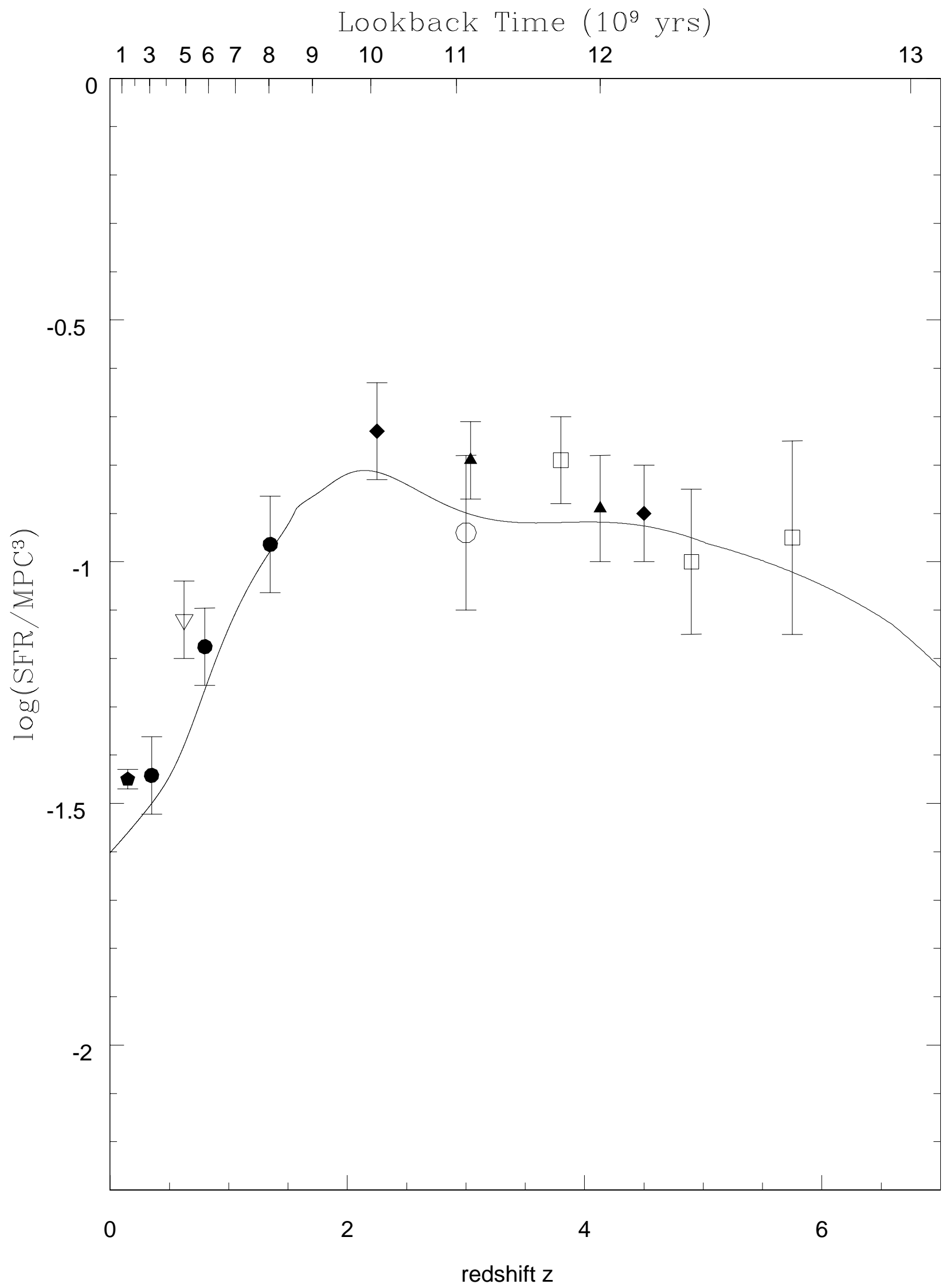




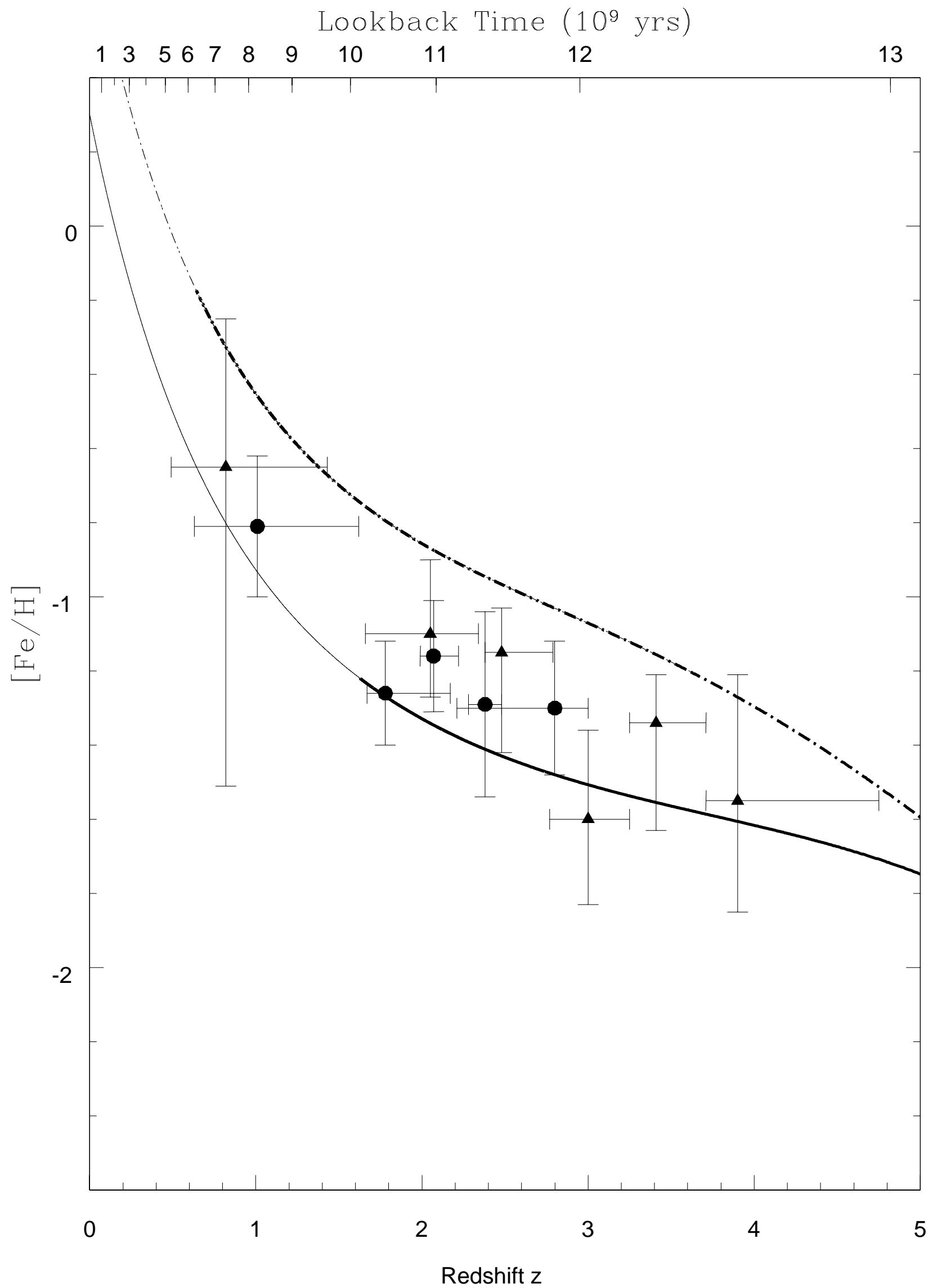

